\title{
Correction to: Comprehensive proteome and phosphoproteome profiling shows negligible influence of RNAlater on protein abundance and phosphorylation
}

Jingi Bae' ${ }^{1}$ Su-Jin Kim', Seung-Eun Lee², Wooil Kwon ${ }^{3}$, Hongbeom Kim³ ${ }^{3}$ Youngmin $\mathrm{Han}^{3}$, Jin-Young Jang ${ }^{3}$, Min-Sik Kim ${ }^{4^{*}}$ (ID and Sang-Won Lee ${ }^{1^{*}}$

\section{Correction to: Clinical Proteomics (2019) 16:18} https://doi.org/10.1186/s12014-019-9239-z In the version of this article that was originally published [1], some information in the "Acknowledgements" section was omitted. This has now been corrected in this Correction note.

\section{Acknowledgements}

This study was supported by the Collaborative Genome Program for Fostering New Post-Genome Industry (NRF-2017M3C9A5031397) and the Brain Research Program (Grant No. NRF-2017M3C7A1027472) through the National Research Foundation (NRF) funded by the Ministry of Science and ICT (MSIT) of Republic of Korea. This work was also supported by the National Research Foundation of Korea (NRF) Grant funded by the Korea government (MSIT) (No. 2019R1C1C1006262). The Biospecimens and data used in this study were provided by the Biobank of Seoul National University Hospital, a member of Korea Biobank Network (SNUH2017-0021).

\section{Author details}

${ }^{1}$ Department of Chemistry, Center for Proteogenome Research, Korea University, Seoul 136-701, Republic of Korea. ${ }^{2}$ Department of Biomedical Science and Technology, Kyung Hee Medical Science Research Institute, Kyung Hee University, Seoul, Republic of Korea. ${ }^{3}$ Department of Surgery and Cancer Research Institute, Seoul National University College of Medicine, Seoul, Republic of Korea. ${ }^{4}$ Department of New Biology, DGIST, Daegu 42988, Republic of Korea.
The original article can be found online at https://doi.org/10.1186/s1201 4-019-9239-z.

Published online: 11 July 2019

\section{Reference \\ 1. Bae J, et al. Clin Proteom. 2019;16:18. https://doi.org/10.1186/s1201 4-019-9239-z.}

\section{Publisher's Note}

Springer Nature remains neutral with regard to jurisdictional claims in published maps and institutional affiliations.

\footnotetext{
*Correspondence: mkim@dgist.ac.kr; sw lee@korea.ac.kr

${ }^{1}$ Department of Chemistry, Center for Proteogenome Research, Korea University, Seoul 136-701, Republic of Korea

${ }^{4}$ Department of New Biology, DGIST, Daegu 42988, Republic of Korea

Full list of author information is available at the end of the article
} 